\title{
Análisis de una Escala para Evaluar la Satisfacción del Profesional Docente en la Enseñanza Primaria
}

\author{
Analysis of a Scale to Evaluate the Satisfaction of the \\ Educational Professional in the Elementary Education
}

Josefa Rodríguez Pulido, Jaime León González-Vélez, María Victoria Aguiar Perera* y Josué Artiles Rodríguez

Universidad de Las Palmas de Gran Canaria

\begin{abstract}
Este trabajo forma parte de un estudio promovido por el grupo de investigación y de innovación pedagógica de la Universidad de Barcelona y el Observatorio Internacional de la Educación de la Profesión Docente. Elaborar una escala de valoración de la satisfacción docente (ESD) es el objetivo de esta investigación, por ello se determina si el instrumento desarrollado y las inferencias realizadas en este cuestionario son válidas. La escala se aplica a una muestra de 215 profesores de primaria en la Comunidad Autónoma de Canarias (Gran Canaria). El cuestionario propuesto por el grupo de FODIP, consta de 18 ítems tipo Likert de 1 a 5 . Los resultados muestran que la escala de valoración aportada es válida y fiable, y que su estructura de tres factores (la planificación, la autonomía y el desarrollo profesional) es una alternativa ágil y rápida para conocer el grado de satisfacción del profesorado. Las tres dimensiones de la escala encuentran correlatos similares en modelos anteriores y, aunque como ya señalamos, el número de dimensiones estimado por los distintos investigadores es variado, a juzgar por las referencias, una escala corta y de fácil aplicación puede ayudar a una primera aproximación a la satisfacción docente.
\end{abstract}

Palabras clave: Satisfacción del profesional docente, Enseñanza primaria, Escala, Planificación, Autonomía, Desarrollo profesional.

This work is part of a study promoted by the Group of research and teaching innovation of the University of Barcelona and the international Observatory of the education of the profession teaching. To elaborate a scale of valuation of the educational satisfaction (ESD) is the aim of this investigation, for it decides if the developed instrument and the inferences that realized in this questionnaire are valid. The scale is applied to a sample of 215 primary teachers in the autonomous community of Canary Islands (Gran Canaria). The questionnaire to assess the satisfaction of professional teachers in primary education proposed by the Group of FODIP, it consists of 18 items Likert-type 1 to 5 . The results show that the scale of valuation provided is valid and reliable, and that its structure of three factors (planning, autonomy and professional development) is an agile and fast alternative to knowing the degree of satisfaction of the teaching staff. The three dimensions of the scale are similar correlates in previous models and, although as already noted, the number of dimensions estimated by different researchers is varied, judging by the references, a scale short and easy application can help a first approach to teacher satisfaction.

Keywords: Satisfaction of the educational professional, Elementary education, Scale, Planning, Autonomy, Professional development.

*Contacto: mariavictoria.aguiar@ulpgc.es issn: 1989-0397

www.rinace.net/riee/

https://revistas.uam.es/riee
Recibido: 18 de octubre de 2015 $1^{\text {a }}$ Evaluación: 30 de enero de 2016 Aceptado: $\quad$ 14 de febrero de 2016 


\section{Introducción}

Elaborar una escala de valoración de la satisfacción docente (ESD) es la aportación de esta investigación. Nuestra contribución viene apoyada por la satisfacción en el trabajo educativo como una dimensión importante de la salud de la organización y desarrollo de los estudiantes. La indagación "del profesor ideal ha sido una constante a lo largo de la historia, su estilo de enseñanza es un valor importante en todo proceso educativo dada la repercusión de la organización del material de estudio, su forma de comunicarse, la directividad de la clase, su rol orientador, consejero, facilitador del aprendizaje y transformador de la cultura, su nivel de control de la clase, su imparcialidad, empatía, entusiasmo y su disponibilidad afectiva. Todas ellas son cualidades personales consideradas como prerrequisitos para el éxito de la enseñanza. No podemos olvidarnos de que el fenómeno educativo, a pesar de sus implicaciones sociales, es un fenómeno individual" Peiteado (2013, p.66). De ahí que la emoción positiva que el profesor tenga en relación con la evaluación de su actividad docente nos podría dar evidencias, del presente y del momento, de su nivel de satisfacción (De Pablos, González y González, 2008).

Estudios realizados por Cuscó y Wells (2009) ponen de manifiesto que la insatisfacción del profesorado, viene dada por la realización de muchas tareas ajenas a la profesión y por no tener condiciones para enseñar.

Este trabajo forma parte de un estudio promovido por el grupo de investigación y de innovación pedagógica de la Universidad de Barcelona y el Observatorio Internacional de la Educación de la Profesión Docente (FODIP). El uso de escalas fiables y válidas es esencial para evaluar la satisfacción del trabajo del profesor. Las investigaciones de satisfacción docente han sido planteadas desde diferentes posicionamientos, se realizan investigaciones sobre la satisfacción laboral del docente, del alumnado, del director e incluso en el marco de la educación infantil, primaria, secundaria. Ello conlleva que la terminología utilizada sea variada (malestar docente, salud mental del profesorado, burnout docente, angustia del enseñante o estrés y ansiedad del profesorado).

Las investigaciones realizadas han profundizado no sólo para mejorar la satisfacción personal, sino también para mejorar los resultados de la organización. Satisfacción es un elemento prioritario para evaluar y mejorar la calidad de los maestros, el proceso de formación y el desarrollo organizativo en general (Anaya y Suárez, 2007). Por otra parte, la investigación sobre la satisfacción también es importante para el desgaste y la separación de los docentes (Skaalvik y Skaalvik, 2011).

Desde los primeros estudios desarrollados por Hoppock (1935), se ha ido avanzando hacia estudios relacionados con la interacción de variables personales y ambientales (Carr, 2007; Cuadra y Veloso, 2010; Zubieta y Susinos, 1992). La satisfacción laboral del profesorado tiene implicaciones en su desempeño docente, por lo que evaluarla adquiere gran importancia para procesos de mejora en centros educativos.

En este contexto puede depender de dos grupos de variables o dimensiones, ambientales y personales, subrayando que los elementos relacionados con dichas dimensiones pueden influir en la satisfacción o la insatisfacción de los docentes. Las variables ambientales agrupan el clima laboral en el centro y el aula (Chaplain, 2008; Klassen y Chiu, 2010), la variabilidad de la actividad laboral, el salario (Butt et al., 2005; Crossman y Harris, 2006), las posibilidades de participación que ofrece la dirección y la aceptación por parte de la comunidad educativa (Greenglass y Burke, 
2003). Las variables personales están relacionadas con la edad y el género (Fraser y Hodge, 2000; Klassen y Chiu 2010; Liu y Ramsey, 2008).

Compartimos con Tejero y Fernández (2009) que la satisfacción laboral es el estado emocional del docente o lo que es lo mismo, la vinculación afectiva al desempeño profesional realizado, ya sea de manera global o en distintas esferas del trabajo (Belkelman, 2004).

La satisfacción profesional de los docentes se compone de una serie de componentes estructurales que permiten la regulación de acciones pedagógicas y el comportamiento en las tareas del profesorado, y es un elemento crucial en la satisfacción en el trabajo docente (Caprara, Barbaranelli, Steca, y Malone, 2006). Por lo tanto, a través de los procesos de formación, todos los aspectos que pueden favorecer la satisfacción profesional se deben tomar en cuenta, entre los que se destacan: el compromiso de la enseñanza (Feather y Rauter, 2004), las condiciones de trabajo, la realización personal a través del desempeño y el bienestar profesional (Nir y Bogler, 2008; Vansteenkiste et al, 2007), el reconocimiento social de la profesión, las políticas educativas que normalizan el desempeño de los docentes y las posibilidades de desarrollo profesional (Hernández, 2014), y los beneficios de la autonomía en la enseñanza (Skaalvik y Skaalvik, 2014). Papel relevante que también tiene la institución, ya que "cuando la institución no respalda, no garantiza las condiciones de realización de la tarea, los sujetos la asumen en soledad, convirtiéndose en individualidades que tratan de afrontar la situación con sus propios medios y recursos personales". (Alliaud y Vezub, 2012, p.950)

Destacar que estudios muestran que la satisfacción laboral del profesorado disminuye progresivamente conforme se avanza en antigüedad profesional, de ahí que los profesores más veteranos manifiestan estar menos a gusto con su trabajo actual y con la vida en el centro docente que sus colegas de menor antigüedad (Nieto y LópezMartín, 2015). E incluso estudios realizados indican la diferencia entre el profesorado según el nivel donde desarrollan la actividad docente. Nieto y Riveiro (2006) los profesores de secundaria aparecen menos satisfechos que los de infantil y primaria. Guerrero (2003) en la universidad los niveles de insatisfacción vienen dados, entre otros, por la falta de apoyo de los compañeros, la indefinición de tareas y por la carencia de recursos.

\section{Modelos y dimensiones vinculados a la satisfacción docente}

Byrne (1999) valida un modelo configurado por cinco dimensiones que condicionan y conforman la satisfacción o insatisfacción docente, pudiendo generar situaciones de estrés laboral y, en determinados casos, burnout en el profesional docente. Según el autor, los factores del modelo hacen referencia al conflicto de rol, la ambigüedad del rol, la carga de trabajo, la labor en el aula, la autonomía, y la relación con compañeros. Siguiendo a este autor, el conflicto de rol es la dimensión vinculada a las expectativas de lo que debería ser el trabajo que realiza y lo que es en realidad; la ambigüedad del rol, está relacionada con la ausencia de protocolos o información clara respecto a su práctica docente, desde el punto de vista administrativo y educativo, en aspectos legales y responsabilidades; la carga de trabajo: es la dimensión relacionada con la cantidad y dificultad de trabajo que pueden llevar a cabo los docentes; la labor en el aula refiere 
aspectos que influyen en la satisfacción del docente por la situación que vive en la clase, como el clima, el rendimiento y comportamiento del alumnado y la disciplina; autonomía es la dimensión que hace referencia a la libertad para tomar decisiones en situaciones de trabajo relacionadas con la práctica docente y el trabajo administrativo de los docentes; por último, la dimensión relación con compañeros, está asociada a la calidad de las interacciones que los docentes tienen con sus compañeros y la dirección del centro.

Respecto a estas últimas dimensiones, autonomía y relaciones, Evans (1999) señala que las interacciones entre el profesional docente y la dirección del centro son un factor determinante en la satisfacción de los docentes, incrementándose el bienestar a medida que los profesionales tenían más participación y estaban más implicados en la gestión, con el consiguiente aumento de la autonomía. Gran parte de la investigación que ha analizado la satisfacción del profesional docente, las motivaciones y el estrés laboral (Brunetti, 2001), tienen un nexo común en la necesidad de autonomía en el trabajo. Los estudios reflejan que la motivación del profesor y la autonomía están relacionadas con la satisfacción en el trabajo y la tensión a la que están sometidos. Los docentes con motivaciones intrínsecas están más satisfechos con sus empleos y son los que menos estrés experimentan (Davis y Wilson, 2000).

Pearson y Moomaw (2006), conscientes de la importancia de la autonomía en la satisfacción docente desarrollan una escala (Teaching Autonomy Scale) para poder evaluar los cuatro factores planteados en su modelo: selección de actividades y materiales, normas de conducta en el aula, la instrucción de planificación y secuenciación de la enseñanza y, por último, toma de decisiones personales en el trabajo.

La Escala de Satisfacción Laboral-Versión para Profesores (ESL-VP; Anaya y Suárez, 2007) evalúa la satisfacción laboral en torno a treinta y dos ítems que configuran cinco dimensiones denominadas: diseño del trabajo, es la dimensión relacionada con la satisfacción laboral en cuanto a la participación de los docentes en la planificación y diseño de objetivos relacionados con su trabajo; condiciones de vida asociadas al trabajo, es la dimensión vinculada con la situación del contexto escolar, la seguridad y los recursos existentes; realización personal conforma la dimensión que hace referencia a la percepción laboral positiva de los docentes en torno a su puesto de trabajo y el sentimiento de adecuación al mismo; promoción y superiores, evalúa las posibilidades de ascenso y la equidad de las personas con rango superior; por último, salario es la dimensión que establece la relación entre satisfacción y el sueldo recibido.

La escala de Anaya y Suárez combina dimensiones relacionadas con motivaciones extrínsecas, como el salario o la promoción y superiores, con dimensiones relativas a la motivación intrínseca, reflejadas en el diseño del trabajo o la realización personal. La satisfacción en el trabajo y la motivación pueden estar influidas por factores externos tales como el salario, la política educativa y las reformas o las condiciones en las que los docentes desempeñan su labor, aunque estos factores no son tan determinantes como los factores del contexto más inmediato al profesorado, relativos a las relaciones o su actividad en el aula (Evans, 1998).

La percepción de la adecuación al puesto de trabajo y el bienestar en el desempeño realizado forman parte de la concepción sobre las competencias que posee el profesorado, siendo un factor de satisfacción que influye en la eficacia en situaciones de estrés del profesorado (Dick y Wagner, 2001; Friedman, 2003). Los docentes que 
poseen una percepción positiva sobre sus capacidades y desempeño tienen una satisfacción laboral mayor y contrarrestan mejor las situaciones problemáticas que aquellos que no se perciben como buenos profesores (Salanova, Cifre, Grau, Llorens y Martínez, 2005).

Satisfacción en el trabajo, a pesar de que es un factor estudiado, requiere una mayor profundización (Anaya y Suárez, 2007), siendo una de las prioridades para diseñar escalas con propiedades psicométricas. Por lo tanto, en esta escala, nos hemos centrado en proporcionar datos que sirvan para asumir la responsabilidad apropiada, como, medidas eficaces pertinentes para su mejora. Este instrumento no se basa en un modelo único, pero reúne los constructos relevantes e ítems de diferentes escalas. El punto común entre los estudios son las dimensiones de las relaciones personales, la planificación, la autonomía y el trabajo en clase (Anaya y Suárez, 2007; Brunetti, 2001; Byrne, 1999; Evans, 1999; Pearson y Moomaw, 2006). Las relaciones personales es sinónimo de la satisfacción con la interacción entre profesores dentro de la escuela (Skaalvik y Skaalvik, 2011). Planificación se refiere a la satisfacción con la programación y ejecución de actividades en la escuela y en casa (Nir y Bogler, 2008). La autonomía se centra en la satisfacción relacionada a la independencia en el desempeño de su trabajo (Skaalvik y Skaalvik, 2014). Por último, se refiere a la satisfacción con la actividad en el aula (Klassen y Chiu, 2010). Por lo tanto, el objetivo de este trabajo es elaborar una escala de satisfacción docente explorando si los datos recogidos con el instrumento, basado en factores comunes propuestos en la literatura científica, son fiables y válidos.

\section{Metodología}

\subsection{Muestra}

La muestra del estudio que describimos está constituida por 215 profesores de ambos sexos y de diferentes centros de una Comunidad Autónoma de España (islas de Gran Canaria y Lanzarote), que imparten docencia en la etapa de educación primaria, lo que representa el $43,26 \%$ del total de profesores que componen el claustro de los centros seleccionados.

\subsection{Instrumento}

El cuestionario para evaluar la satisfacción del profesional docente en la Enseñanza Primaria (ESEP) propuesto por el grupo de FODIP (2013), aplicado en el curso 20132014, consta de 18 ítems tipo Likert de 1 (nada satisfecho) a 5 (muy satisfecho) desarrollados con el objetivo de indagar el nivel de satisfacción de los profesionales en educación primaria. El análisis de la profesión docente obliga a explorar su actividad profesional desde los diferentes componentes estructurales que la conforman, es decir, las características de los sujetos que la realizan (su preparación, intereses, motivos), las condiciones en que tiene lugar el proyecto educativo, los métodos, las vías y los medios que se emplean para su desarrollo, así como las particularidades de los estudiantes en los que recae la acción pedagógica de los profesores y las políticas curriculares que enmarcan este proceso.

\subsection{Procedimiento}

En primer lugar calculamos los estadísticos descriptivos, media, desviación típica, asimetría y curtosis, de cada ítem. A continuación, para determinar la estructura factorial de la escala utilizamos la técnica multivariada, modelo de ecuaciones 
estructurales exploratorio (MESE), desarrollado por Asparouhov y Muthen (2009). La ventaja principal de esta técnica es combinar el análisis factorial exploratorio (AFE) con el análisis factorial confirmatorio (AFC), además no requiere que el peso factorial de los ítems sea cero en los factores no correspondientes, por lo que el cálculo de los índices de ajuste y de las correlaciones entre variables latentes es más preciso (Asparouhov y Muthén, 2009; Marsh et al., 2009).

Respecto al método de rotación utilizado, siguiendo las recomendaciones de Asparouhov y Muthen (2009) para situaciones en las que se conoce poco de la estructura factorial a estudiar utilizamos una rotación geomin. Más especificamente, utilizamos el geomin oblícuo, ya que los métodos que implican rotaciones oblicuas, en las ciencias sociales, muestran relaciones entre factores más cercanas a la realidad (Brown, 2006; Schmitt, 2011).

En cuanto al método de estimación utilizado, al estar la escala formada por ítems tipo Likert, las variables observables son categóricas ordinales (Flora y Curran, 2004) y, por lo tanto, para estimar el valor de los parámetros y los índices de ajuste es más preciso utilizar un método de estimación que no requiere normalidad multivariada (Schmitt, 2011). Por ello, utilizamos el método de mínimos cuadrados ponderados ajustado por la media y la varianza (WLSMW).

Además el hecho de que los profesores estén agrupados por colegios viola el supuesto de independencia, esto puede inflar el valor de $\chi^{2}$ e infraestimar los errores típicos (Stapleton, 2006). Para corregir esto, los parámetros fueron estimados maximizando una función logarítmica ponderada y los errores típicos utilizando un estimador tipo sandwich (Muthén y Muthén, 1998-2014). Para analizar el ajuste del modelo al patrón de los datos utilizamos la prueba de $\chi^{2}$, la ratio $\chi^{2} /$ g.l., el índice RMSEA y su intervalo con un nivel de cofianza del 90\%, el índice de Tucker-Lewis (TLI), el índice de ajuste comparativo (CFI) y el residuo ponderado cuadrático medio (WRMR).

Para decidir el número de factores seguimos varios criterios, en primer lugar el sentido teórico de los mismos, en segundo lugar, siguiendo las recomendaciones de Costello y Osborne (2005), se debe elegir una estructura factorial en la que haya más de tres ítems por factor y con el menor número de pesos factoriales cruzados, por lo que en este estudió se prestó atención al número de factores con al menos tres ítems significativos en el factor esperado y que fueran significativos, con un nivel de confianza del 95\%, únicamente en uno o dos factores y, por último, se atendió a los índices de ajuste, teniendo en cuenta que seguir este criterio puede llevar a aceptar más factores de los necesarios (Hayashi, Bentler y Yuan, 2007). Una vez determinado el número de factores, para evitar pesos factoriales cruzados se descartaron los ítems con pesos superiores a .35 en dos o más factores, luego se volvió a analizar la estructura factorial, así hasta que no hubiera ítems con pesos cruzados superiores a .35.

A continuación, se analizaron las evidencias de fiabilidad, para ello utilizamos el omega ( $\omega)$ de McDonald (1999) a partir de la matriz de correlaciones policóricas, ya que este índice no requiere que el peso factorial sea igual para todos los ítems (Yang y Green, 2010) ni que los datos sean continuos (Elosua y Zumbo, 2008), al igual que con el alfa de Cronbach con valores por encima de .85 se considera que la escala es fiable. Los valores perdidos se estimaron utilizando un método imputación multivariante mediante ecuaciones encadenadas a través de la librería mice 2.9 (Buuren y GroothuisOudshoorn, 2011) del software R (R Core Development Team, 2014). Para calcular los estadísticos descriptivos se utilizó el software R; para el MESE, el Mplus 7.11 (Muthén 
y Muthén, 1998-2014) y, por último, para estimar el omega de McDonald se utilizó la librería psych (Revelle, 2013) del programa estadístico R.

\section{Resultados}

\subsection{Análisis descriptivos}

En primer lugar se calcularon los estadísticos descriptivos de cada ítem. Como se puede observar en la Tabla 1, la media osciló entre 3.023 para el ítem 18 y 4.171 para el ítem 10, la desviación típica entre .711 y 1.123, ítems 15 y 18 respectivamente. En cuanto a la asimetría y la curtosis, los valores estuvieron por debajo de 2 en valores absolutos, es decir, valores bajos.

Tabla 1. Media, desviación típica y pesos factoriales de los ítems finales

\begin{tabular}{|c|c|c|c|c|c|}
\hline & MEDIA & D.T. & $\mathbf{P .}_{.1}$ & A. 2 & Pr.s \\
\hline \multicolumn{6}{|l|}{ Planificación } \\
\hline $\begin{array}{l}\text { 1. Satisfacción del profesorado para implicarse en el } \\
\text { proyecto educativo de la escuela }\end{array}$ & 3,89 &, 74 & ,39 & ,28 & ,26 \\
\hline $\begin{array}{l}\text { 2. Satisfacción del profesorado para participar en } \\
\text { actividades extraescolares }\end{array}$ & 3,80 &, 91 & ,79 &,- 01 & ,02 \\
\hline $\begin{array}{l}\text { 3. Satisfacción del profesorado para participar en } \\
\text { actividades externas establecidas por la escuela }\end{array}$ & 3,71 & ,92 &, 90 & ,01 &,- 01 \\
\hline \multicolumn{6}{|l|}{ Autonomía } \\
\hline $\begin{array}{l}\text { 4. Satisfacción del profesorado para tomar } \\
\text { iniciativas propias }\end{array}$ & 3,94 &, 87 &, 12 & ,45 & ,25 \\
\hline $\begin{array}{l}\text { 5. Satisfacción del profesorado para trabajar en } \\
\text { equipo con los colegas }\end{array}$ & 4,00 &, 84 &,- 14 & ,82 &,- 01 \\
\hline $\begin{array}{l}\text { 6. Satisfacción del profesorado para relacionarse con } \\
\text { otros colectivos de la escuela (comunidad, familia, } \\
\text { etc.) }\end{array}$ & 3,88 & ,84 &,- 01 & ,64 & ,23 \\
\hline $\begin{array}{l}\text { 7. Satisfacción del profesorado para la realización } \\
\text { del plan de acción tutorial establecido por la } \\
\text { escuela }\end{array}$ & 3,80 &, 84 & ,04 & ,70 &, 14 \\
\hline $\begin{array}{l}\text { 8. Satisfacción del profesorado para implicarse en } \\
\text { los procesos de comunicación de la escuela }\end{array}$ & 3,73 & ,86 &,- 03 &, 90 & ,06 \\
\hline $\begin{array}{l}\text { 9. Satisfacción del profesorado para formar parte del } \\
\text { equipo directivo u órganos de gestión de la } \\
\text { escuela }\end{array}$ & 3,57 & 1,09 & ,20 &, 52 &,- 08 \\
\hline \multicolumn{6}{|l|}{ Desempeño } \\
\hline $\begin{array}{l}\text { 10. Satisfacción para la realización de su labor } \\
\text { docente }\end{array}$ & 4,09 &, 71 & ,02 &, 10 &, 71 \\
\hline $\begin{array}{l}\text { 11. Satisfacción en cuanto al proceso de enseñanza- } \\
\text { aprendizaje en el aula }\end{array}$ & 4,03 &, 71 &,- 03 & ,0O & ,98 \\
\hline
\end{tabular}

Notas. 1: Planificación; 2: Autonomía; 3: Profesional.

Fuente: Elaboracion propia.

\subsection{Estructura factorial}

En la Tabla 2 se puede observar que en la estructura factorial de 4 factores coincide el número de factores con tres o más ítems significativos en un factor y que no son significativos en más de uno o dos factores. Respecto a los índices de ajuste, se puede observar que a mayor número de factores, mejor ajuste, datos que están en consonancia con los resultados de Hayashi et al. (2007). Asparouhov y Muthen (2009) han observado, con puntuaciones continuas, que si añadir un factor disminuye el SRMR en $.001 \mathrm{o}$ menos, no merece la pena mantener la estructura con más factores. En este estudio, al ser los ítems variables categóricas se atendió al valor del RMSEA y así, a 
raíz de los datos, podríamos descartar la opción de más de cinco factores. Finalmente, atendiendo a la discriminación de los factores y al valor de RMSEA se optó por la estructura de 4 factores. A continuación se eliminaron cuatro ítems por tener pesos superiores a .35 en dos o más factores, seguidamente se analizó la estructura factorial sin estos ítems y observamos que tres ítems tenían pesos superiores a .35 en dos factores, así que los descartamos y en el siguiente análisis de la estructura factorial se observó que un factor quedaba solo con un ítem, por lo que se decidió por una estructura factorial de tres factores, donde el primer factor, planificación, determinado por 3 ítems; el segundo factor, autonomía, formado por 6 ítems; y, finalmente, el tercer factor, desempeño docente, formado, únicamente, por 2 ítems. Esta estructura factorial cuenta con adecuados índices de ajuste $\chi^{2}(214,33)=109,15(p=0,00)$, RMSEA $=0,10$ $[0,08 ; 0,10] \mathrm{CFI}=0,96, \mathrm{TLI}=0,91$, and $\mathrm{WRMR}=0,59 \mathrm{y}$ con pesos factoriales en sus respectivos factores que oscilaron entre 0,392 y 0,976 (Ver Tabla 1).

Tabla 2. Número de factores con tres ítems significativos (95\%) en un factor y que no son significativos en más de uno o dos factores e índices de ajuste en función del número de factores en cada extracción

\begin{tabular}{lcccccccc}
\hline FACTORES & FACT/ÍtEMS & $\mathbf{X}^{\mathbf{2}}$ & G.L. & RMSEA & $\mathbf{9 0} \%$ & CFI & TLI & WRMR \\
\hline 1 & 1 & 396.026 & 135 & .093 & $.083-.104$ & .905 & .893 & 1.291 \\
2 & 2 & 30.309 & 118 & .083 & $.072-.095$ & .934 & .914 & .966 \\
3 & 2 & 236.337 & 102 & .077 & $.064-.090$ & .951 & .927 & .748 \\
4 & 4 & 181.945 & 87 & .700 & $.056-.084$ & .966 & .939 & .575 \\
5 & 3 & 122.621 & 73 & .055 & $.038-.072$ & .982 & .962 & .406 \\
6 & 4 & 103.560 & 60 & .057 & $.038-.075$ & .984 & .960 & .351 \\
\hline
\end{tabular}

Notas: Fact/Ítems =Número de factores que tienen al menos 3 ítems significativos en el factor que más pesan y no son significativos en más de dos factores $(\mathrm{NC}=95 \%)$.

Fuente: Elaboracion propia.

\subsection{Fiabilidad}

Los resultados mostraron una fiabilidad adecuada tanto de la escala global de 11 ítems, $\omega_{\mathrm{T}}=0,95$, como de las 3 dimensiones: planificación $(\omega=0,87)$, autonomía $(\omega=0,89)$ y desempeño docente $(\omega=0,87)$.

\section{Discusión y conclusiones}

Como se puede observar en la introducción, las escalas sobre satisfacción laboral tienen distintas dimensiones aunque algunas de ellas se reiteran a lo largo de la literatura ad $h o c$, si no en su continente, sí en su contenido.

El objetivo de este estudio ha sido elaborar una escala de satisfacción docente, examinado las propiedades psicométricas de la escala desarrollada por el grupo FODIP (2013). En primer lugar se analizó la estructura factorial de la escala y, a continuación, se exploró la fiabilidad de la misma. Los resultados mostraron que la estructura factorial más apropiada es la de tres factores (planificación, autonomía y desempeño docente), al proporcionar un mejor ajuste, observando adecuadas evidencias de validez y fiabilidad.

Así, la dimensión planificación, con una adecuada fiabilidad $(\omega=0,87)$, guarda relación con el denominado diseño del trabajo, en la escala de Anaya y Suárez (ESL-VP), una dimensión estrechamente relacionada con la participación, que otorga protagonismo a la implicación del profesorado en el diseño de los objetivos relativos a su encargo docente. La dimensión autonomía, en sus distintas concepciones, presentada sola o 
acompañada de algún otro componente, constituye una de las categorías más analizada (Zubieta y Susinos, 1992; Evans, 1999; Byrne, 1999; Brunetti, 2001; Pearson y Moomaw, 2006). En este estudio, esta dimensión también presenta una fiabilidad $(\omega=.89)$.

Por último, la dimensión desempeño docente refiere al trabajo del profesorado en el aula, en el desarrollo del proceso de enseñanza-aprendizaje. Es en relación con esta dimensión, en la esfera más próxima a su quehacer cotidiano como docente, donde el profesorado muestra un mayor grado de satisfacción. Byrne (1999) contempla la labor en el aula como una de las cinco dimensiones del modelo que propone; Anaya y Suárez (2007) incluyen la realización personal como una dimensión que alude a una percepción positiva del desempeño laboral del profesorado, existiendo evidencias sobre la relación entre una percepción positiva del desempeño laboral y un mejor manejo del estrés (Dick y Wagner, 2001; Friedman, 2003). Igualmente, los efectos del afrontamiento de situaciones problemáticas se ven amortiguados cuando existe una satisfacción con el desempeño profesional del profesorado (Salanova, Cifre, Grau, Llorens y Martínez, 2005).

Como puede apreciarse, las tres dimensiones de la escala encuentran correlatos similares en modelos anteriores $\mathrm{y}$, aunque como ya señalamos, el número de dimensiones estimado por los distintos investigadores es variado, a juzgar por las referencias al respecto, una escala corta y de fácil aplicación puede ayudar a realizar una primera y sencilla aproximación a la satisfacción docente en los centros de educación primaria.

La escala resultante constituye un instrumento rápido y fácil en su manejo que puede contribuir a la realización de un primer diagnóstico del funcionamiento de un centro al evaluar la satisfacción de los docentes, ya que posee unas adecuadas propiedades psicométricas.

Como conclusión final, destacar que la escala constituye un instrumento rápido y fácil en su manejo y, al poseer unas adecuadas propiedades psicométricas, puede contribuir a la realización de un primer diagnóstico del funcionamiento de un centro al evaluar la satisfacción de los docentes.

\subsection{Recomendaciones}

En esta investigación tan solo se ha realizado un análisis preliminar de la escala diseñada por el grupo FODIP, por lo tanto, en futuras investigaciones sería recomendable, analizar las propiedades psicométricas de la escala con una muestra más extensa, si bien es cierto que se ha observado que se puede trabajar adecuadamente con muestras alrededor de los 200 sujetos como se ha reflejado en otros trabajos (Albaladejo-Blázquez, Ferrer-Cascales, Reig-Ferrer y Fernández-Pascual, 2013; Ruiz, Pardo y San Martín, 2010).

Por último, Fraser y Hodge (2000), plantean que variables personales como la edad puedan influir en la satisfacción laboral de los docentes, por lo tanto sería interesante analizar si los profesores con experiencia docente interpretan el cuestionario de forma similar a profesores con menos años de docencia. 


\section{Referencias}

Albaladejo-Blázquez, N., Ferrer-Cascales, R., Reig-Ferrer, A. y Fernández-Pascual, M. (2013). Does school violence occur in preschool and primary education? An assessment and management proposal. Anales de Psicología, 29(3), 1060-1069. doi: 10.6018/analesps.29.3.158431.

Alliaud, A. y Vezub, L. (2012). El oficio de enseñar. Sobre el quehacer, el saber y el sentir de docentes argentinos. Revista Diálogo Educativo, 12(37), 927-952. doi:10.7213/dialogo.educ.7211

Anaya, D. y Suárez, J. M. (2007). Job satisfaction of Spanish teachers in preschool, primary and secondary education levels. A study within the national scope. Revista de Educación, 344, 217-243.

Asparouhov, T. y Muthén, B. (2009). Exploratory structural equation modeling. Structural Equation Modeling, 16(3), 397-438. doi:10.1080/10705510903008204.

Belkelman, S. (2004). Job Satisfaction. CQ Weekly, 62(40), 2420-2423.

Brown, B. M. (2006) Confirmatory factor analysis for applied research. Nueva York: Guilford.

Brunetti, G. (2001). Why do they teach? A study of job satisfaction among long-term high school teachers. Teacher Education Quarterly, 28(2), 49-74.

Butt, G., Lance, A., Fielding, A., Gunter, H., Rayner, S. y Thomas, H. (2005). Teacher job satisfaction: Lessons from the TSW pathfinder project. School Leadership and Management, 25(5), 455-471. doi:10.1080/13634230500340807.

Buuren, S. y Groothuis-Oudshoorn, K. (2011). MICE: Multivariate imputation by chained equations in R. Journal of Statistical Software, 45(3), 1-67. doi:10.18637/jss.v045.io3.

Byrne, B. (1999). The nomological network of teacher burnout: A literature review and empirically validated model. En R. Vandenberghe y A. Huberman (Eds.), Understanding and preventing teacher burnout (pp. 15-37). Cambridge: Cambridge University Press.

Caprara, G., Barbaranelli, C., Steca, P. y Malone, P. (2006). Teachers' self-efficacy beliefs as determinants of job satisfaction and students' academic achievement: A study at the school level. Journal of School Psychology, 44(6), 473-490. doi:10.1016/j.jsp.2006.09.001.

Carr, A. (2007). Psicología Positiva. La ciencia de la felicidad. Barcelona: Paidós.

Chaplain, R. P. (2008). Stress and psychological distress among trainee secondary teachers in England. Educational Psychology, 28(2), 195-209. doi:10.1080/01443410701491858.

Costello, A. y Osborne, W. (2005). Best practices in exploratory factor analysis: Four recommendations for getting the most from your analysis. Practical Assesment Research $y$ Evaluation, 10(4), 1-9.

Crossman, A. y Harris, P. (2006). Job satisfaction of secondary school teachers. Educational Management Administration and Leadership, 34(1) 29-46. doi:10.1177/1741143206059538.

Cuadra, A. y Veloso, C. (2010). Degree of supervision like moderating variable between the leadership and satisfaction, motivation and organizational climate. Revista Chilena de Ingeniería, 18(1), 15-25. doi:10.4067/s0718-33052010000100003.

Cuscó, M. B. I. y Wells, J. G. (2009). La satisfacción del profesor de Educación Física. Educación Física y Deporte, 27(2), 27-35.

Davis, J. y Wilson, S. (2000). Principals' efforts to empower teachers: Effects on teacher motivation and job satisfaction and stress. The Clearing House, 73(6), 349-357. doi:10.1080/00098650009599442 
De Pablos, J., González, T. y González, A. (2008). El bienestar emocional del profesorado en los centros TIC como factor de innovación educativa. Revista Latinoamericana de Tecnología Educativa, 7(2), 45-55.

Dick, R. y Wagner, U. (2001). Stress and strain in teaching: A structural equation approach. British Journal of Educational Psychology, 71(2), 243-259. doi:10.1348/000709901158505.

Elousa P. y Zumbo, B. (2008). Coefficients of reliability for scales of categorical tidy response. Psicothema, 20, 896-901.

Evans, L. (1998). Teacher morale, job satisfaction and motivation. Londres: Chapman.

Evans, L. (1999). Managing to motivate: A guide for school leaders. Londres: Cassell.

Feather, N. y Rauter, K. (2004). Organizational citizenship behaviors in relation to job status, job insecurity, organizational commitment and identification, job satisfaction, and work values. Journal of Occupational and Organizational Psychology, 77(1), 81-94. doi:10.1348/096317904322915928.

Flora D. y Curran P. J. (2004). An empirical evaluation of alternative methods of estimation for confirmatory factor analysis with ordinal data. Psychological Methods, 9(4), 466-491. doi:10.1037/1082-989x.9.4.466.

Fraser, J. y Hodge, M. (2000). Job satisfaction in higher education: Examining gender in professional work settings. Sociological Inquiry, $7 O(2)$, 172-178. doi:10.1111/j.1475$682 \times$.2000.tbo0904.x.

Friedman, I. (2003). Self-efficacy and burnout in teaching: The importance of interpersonal relations efficacy. Social Psychology of Education, 6(3), 191-215.

Guerrero, E. (2003). Análisis pormenorizado de los grados de burnout y técnicas de afrontamiento del estrés docente en profesorado universitario. Anales de Psicología, 19(1), 145-158.

Greenglass, E. y Burke, R. (2003). Teacher stress. En M. Dollard, H. R. Winefield y A. H. Winefield (Eds.), Occupational stress in the service professions (pp. 213-236.). Nueva York: Taylor and Francis.

Hayashi, K., Bentler, P. y Yuan, K. (2007). On the likelihood ratio test for the number of factors in exploratory factor analysis. Structural Equation Modeling, 14(3), 505-526. doi:10.1080/10705510701301891

Hernández, A. (2014). La "Satisfacción Profesional": premisa o resultado del desarrollo profesional del profesorado. En T. Mentado y L. Castro (Coord.), Satisfacción Profesional del profesorado de Educación Primaria. VI Seminario Internacional del Observatorio Internacional de la Profesión Docente (pp. 13-25). Barcelona: Universidad de Barcelona.

Hoppock, R. (1935.) Job satisfaction. Nueva York: Arno Press.

Klassen, R. y Chiu, M. (2010). Effects on teachers' self-efficacy and job satisfaction: Teacher gender, years of experience, and job stress. Journal of Educational Psychology, 102(3), 741756. doi:10.1037/a0019237.

Liu, X. y Ramsey, J. (2008). Teachers' job satisfaction: Analyses of the Teacher Follow-Up Survey in the United States for 2000-2001. Teaching and Teacher Education, 24(5), 11731184. doi:10.1016/j.tate.2006.11.010.

Marsh, H., Muthén, B., Asparouhov, T., Lüdtke, O., Robitzsch, A., Morin, A. y Trautwein, U. (2009). Exploratory structural equation modeling, integrating CFA and EFA: Application to students' evaluations of University Teaching. Structural Equation Modeling, 16(3), 439-476. doi:10.1080/10705510903008220 
McDonald, R. (1999). Test theory: A unified treatment. Mahwah, NJ: Lawrence Erlbaum Associates.

Muthén, L. y Muthén B. (1998-2014). Mplus User’s Guide. Los Ángeles, CA: Muthén \& Muthén.

Nieto, D. A. y López-Martín, E. (2015). Satisfacción laboral del profesorado de educación secundaria. Revista de Investigación Educativa, 33(2), $\quad$ 435-452. doi:10.6018/rie.33.2.202841.

Nieto, D. A. y Riveiro, J. M. S. (2006). La satisfacción laboral de los profesores en función de la etapa educativa, del género y de la antigüedad profesional. Revista de investigación educativa, 24(2), 521-556.

Nir, A. y Bogler, R. (2008). The antecedents of teacher satisfaction with professional development programs. Teaching and Teacher Education, 24(2), 377-386. doi: $10.1016 /$ j.tate.2007.03.002

Pearson, L. y Moomaw, W. (2006). Continuing validation of the teaching autonomy scale. The Journal of Educational Research, 100(1), 44-51. doi:10.3200/joer.100.1.44-51.

Peiteado, M. G. (2013). Los estilos de enseñanza y aprendizaje como soporte de la actividad docente. Journal of Learning Styles, 6(11), 70.

R Development Core Team. (2008). R: A language and environment for statistical computing. Viena: R Foundation for Statistical Computing.

Revelle, W. (2013). Psych: Procedures for personality and psychological research. Evanston, IL: Northwestern University.

Ruiz, M. A., Pardo, A. y San Martín, R. (2010). Structural equation models. Papeles del Psicólogo, $31(1), 34-45$.

Salanova, M., Cifre, E., Grau, R., Llorens, S. y Martínez, M. (2005). Precedents of the autoefficiency in teachers and university students: A causal model. Revista de Psicología del Trabajo y de las Organizaciones, 21(1-2), 159-176.

Schmitt T. (2011). Current methodological considerations in exploratory and confirmatory factor analysis. Journal of Psychoeducational Assessment, 29(4), 304-321. doi:10.1177/0734282911406653.

Skaalvik, E. y Skaalvik, S. (2011). Teacher job satisfaction and motivation to leave the teaching profession: Relations with school context, feeling of belonging, and emotional exhaustion. Teaching and Teacher Education, 27(6), 1029-1038. doi:10.1016/j.tate.2011.04.001

Skaalvik, E. y Skaalvik, S. (2014). Teacher self-efficacy and perceived autonomy: Relations with teacher engagement, job satisfaction, and emotional exhaustion. Psychological Reports, 114(1), 68-77. doi:10.2466/14.02.pro.114k14wO

Stapleton, L. (2006). An assessment of practical solutions for structural equation modeling with complex sample data. Structural Equation Modeling, 13(1), 28-58. doi:10.1207/s15328007sem1301_2.

Tejero, C. M. y Fernández, M. J. (2009). Measurement of the labor satisfaction in the school direction. Revista Electrónica de Investigación y Evaluación Educativa, 15(2), 1-15.

Vansteenkiste, M., Neyrinck, B., Niemiec, C. P., Soenens, B., De Witte, H. y Van den Broeck, A. (2007). On the relations among work value orientations, psychological need satisfaction and job outcomes: A self-determination theory approach. Journal of Occupational y Organizational Psychology, 80(2), 251-277. doi:10.1348/096317906x 111024. 
Revista Iberoamericana de Evaluación Educativa

Yang, Y. y Green, S. (2010). A note on structural equation modeling estimates of reliability. Structural Equation Modeling: A Multidisciplinary Journal, 17(1), 66-81. doi:10.1080/10705510903438963.

Zubieta, J. y Susinos, T. (1992). Las satisfacciones e insatisfacciones de los enseñantes. Madrid: CIDE. 